\title{
The relationship between health literacy and general health status of patients with type II diabetes
}

\author{
Milad Borji, ${ }_{1}^{1}$ Asma Tarjoman, ${ }^{2}$ Masoumeh Otaghi, ${ }_{1}{ }^{*}$ Zeynab Khodarahmi ${ }^{2}$ and Ali Sharifi ${ }^{3}$ \\ ${ }^{1}$ Department of Nursing, Faculty of Nursing and Midwifery, Ilam University of Medical Sciences Ilam Iran \\ ${ }^{2}$ Student Research Committee Ilam University of Medical Sciences Ilam Iran \\ ${ }^{3}$ Assistant Professor, Department of Internal Medicine, School of Medicine, Ilam University of Medical \\ Sciences, Ilam Iran
}

\begin{abstract}
Individuals with low health literacy are less aware of their own health status and receive fewer preventive services. Furthermore, fewer chronic diseases are controlled in these individuals. This study aimed to investigate the relationship between health literacy and general health status of patients with type II diabetes.In this descriptive-correlational study, 250 patients with type II diabetes in Ilam were selected using convenience sampling. Tools used in this study were the Test of Functional Health Literacy in Adults (TOFHLA) and General Health Questionnaire-28 (GHQ28). Data analysis was conducted using the SPSS Software version 20, including t-test (for two groups of variables), ANOVA (for more than two groups of variables), and correlation analysis.The results showed that 80 (32\%), 102 (40.8\%), and $68(27.2 \%)$ patients with diabetes had inadequate, marginal, and adequate literacy, respectively. The findings also indicated that the means and standard deviations of patients' health literacy scores were $31.38 \pm 6.40$ in terms of calculations, $34.93 \pm 7.45$ in reading skill, and $6.30 \pm 11.61$ in general. There was a statistically significant relationship between health literacy and general health status of patients $(\mathrm{p}<0.001)$. Based on the results revealing average/ marginal health literacy and general health in most patients in the study, officials must conduct more research to improve health literacy and general health of patients.
\end{abstract}

KEY WORDS: HEALTH LITERACY, DIABETES, GENERAL HEALTH

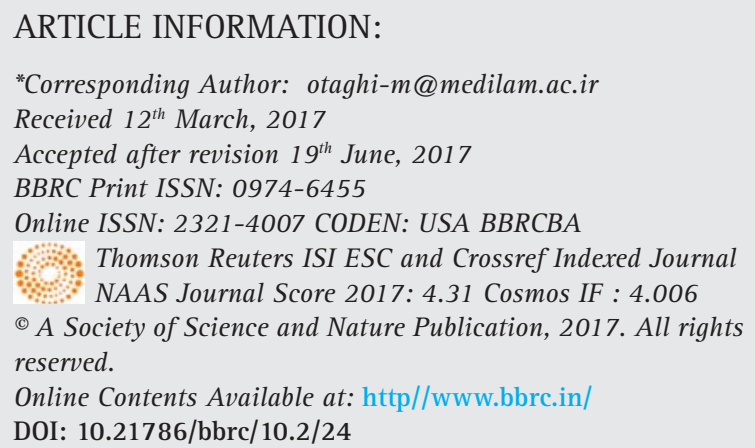




\section{INTRODUCTION}

Health literacy is individual's capacity for process, obtain and understand basic about health information $\&$ services and is necessary for good health (Kindig, Panzer et al. 2004). Health literacy includes reading, listening, analysis, and decision-making skills and the ability to apply these skills in health positions, which is not necessarily associated with education level or general reading inability (Sihota and Lennard 2004). Inadequate health literacy correlates with poorer individual health status report, misuse of drugs and failure to comply with physician's orders, poorer blood sugar control and increased prevalence of individual reports of problems induced by poor control, lower health knowledge, lower contribution in deciding on treatment, less expression of health concerns, and worse relationship with physicians(Kindig, Panzer et al. 2004; Javadzade, Sharifirad et al. 2012) .

Individuals with low health literacy are less aware of their health status, receive fewer preventive services, are under less control for chronic diseases, have poorer physical and mental health performance, and make greater use of the emergency department and hospital services (Peerson and Saunders 2009). Despite the critical importance of identifying individuals with inadequate health literacy, healthcare systems' employees are often less concerned about this issue. In contributing patients with inadequate health literacy, particular methods should be used, including using simple and understandable words and expressions, using images, getting feedback from individuals after providing information to them, and limiting the information provided to the individual at any meeting (Chew, Bradley et al. 2004).

Low level of health literacy is more common among the elderly, illiterate individuals, immigrants, individuals with low mental health, and those with hypertension and type II diabetes. Low health literacy also causes increased mortality, decisions on certain health risk behaviors, and failure to perform preventive behaviors such as screening tests and, thus, poor physical health (Williams, Baker et al. 1998; Kalichman, Benotsch et al. 2000; Kalichman and Rompa 2000; Schillinger, Grumbach et al. 2002; Kindig, Panzer et al. 2004). Health literacy in chronic patients such as patients with diabetes who require self-care plays an essential role, which is why attention to the issue of health literacy in patients with diabetes has been growing in importance (Khosravi, Ahmadzadeh et al. 2015).

Chronic diseases affect patients for many years. For this reason, as long as these conditions are not properly managed and controlled, no further healthcare services are received, which leads to reduced quality of life and health (Esmaeili Shahmirzadi, Shojaeizadeh et al. 2012). Psychological aspects of diabetes have attracted atten- tion of many experts in this area as this disease leads to many behavioral problems in patients. Psychological factors associated with quality of life can have a great impact on the quality of patients' lives. Accordingly, the results of previous studies in this area indicate that mood factors are involved in the prevention of diabetes in patients with diabetic retinopathy (McDarbyc and Acerinie 2014; Moayedi, Zare et al. 2015; Seyedoshohadaee, Kaghanizade et al. 2016).

Given the increasing prevalence of diabetes (Mohan, Sandeep et al. 2007; Seyedoshohadaee, Kaghanizade et al. 2016; Varvani Farahani, Rezvanfar et al. 2016), the present study was conducted in 2015 with the aim to determine the relationship between health literacy and general health status of patients with type II diabetes in Ilam City.

\section{MATERIAL AND METHODS}

In this descriptive-correlational study, according to previous studies conducted in the field (Seyedoshohadaee, Kaghanizade et al. 2016), 250 individuals with diabetes in Ilam City participated in the study. The inclusion criteria were residence in the city of Ilam, ability to read and write, having type II diabetes, and lack of known mental disorders. In this study, the participants were selected using convenience sampling; accordingly, the researcher went to the Shahid Mostafa Khomeini and Imam Khomeini hospitals in Ilam City every morning and gave the questionnaire to diabetes patients who met the inclusion criteria. The questionnaires were completed through self-report.

To collect the health literacy data, the Persian version of the Test about Functional Health Literacy in Adults (TOFHLA) was used, which was previously validated by Tehrani Bani Hashemi et al. (Tehrani Banihashemi, Amirkhani et al. 2007). The questionnaire consists of two parts of computation and reading comprehension. The reading comprehension part has 50 items and examines patients' ability in reading authentic healthcare texts. The computation part evaluates patients' ability to understand and act based on the recommendations given to them by physicians and healthcare educators, which require computation. This part contains 10 health instructions or orders on prescribed drugs, time to go to the doctor, stages of use of grants, and an example of the result of a medical test. Each item in the reading comprehension part was given 1 point (a total of 50 points), and the items (a total of 17) in the computation part were given a total of 50 points by multiplying coefficients for an overall of 100 points for the items in the questionnaire. Based on the point of separation of 59 and 74, the participants' health literacy level was 


\begin{tabular}{|l|l|l|}
\hline \multicolumn{3}{|l|}{ Table 1. Demographic characteristics of patients } \\
\hline $\begin{array}{l}\text { Demographic } \\
\text { features }\end{array}$ & Sub-Categories & $\mathrm{N}(\%)$ \\
\hline \multirow{4}{*}{ Gender } & Male & $107(42.8)$ \\
\cline { 2 - 3 } & Female & $143(57.2)$ \\
\hline \multirow{4}{*}{ Education } & Married & $153(61.2)$ \\
\cline { 2 - 3 } & illiterate & $97(38.8)$ \\
\hline \multirow{4}{*}{ income } & Below Diploma & $54(21.6)$ \\
\cline { 2 - 3 } & Diploma & $141(56.4)$ \\
\cline { 2 - 3 } & Collegiate & $55(22)$ \\
\hline & Less than 500 thousand Rials & $92(36.8)$ \\
\cline { 2 - 3 } & 500 to 1 million & $116(46.4)$ \\
\cline { 2 - 3 } & More than 1 million & $42(16.8)$ \\
\hline
\end{tabular}

classified into three levels of inadequate, marginal, and adequate.

The points were considered based on the proposal by the instrument designers (Tehrani Banihashemi, Amirkhani et al. 2007). The reliability of this questionnaire was computed as 0.79 for the computation part and 0.88 for the comprehension part, in the study by Javadzade on the elderly (Javadzade, Sharifirad et al. 2012)The General Health Questionnaire-28, designed and developed in 1972 by Goldberg, was used to measure general health status. The questionnaire encompasses the following four dimensions: physical health (items 1-7) \&t anxiety and insomnia (items 8-14) \&t social dysfunction (items 15- 21) Et depression (items 22-28). Each item in the questionnaire was accompanied by a 4-point Likert-type response scale consisting of "seldom" (0), "usually" (1), "almost more than usual" (2), and "often more than usual" (3) (Seyedoshohadaee, Kaghanizade et al. 2016). The minimum scores(0) and maximum scores were ( 84)

For each subscale, The minimum scores (0) and maximum scores were (21). Regarding subscales, the scores 14-21, 6-13, and $<5$ were considered indicators of inadequate, average, and adequate general health status, respectively. On the other hand, general health scores of 57-84, 24-56, and <24 were also considered inadequate, average, and adequate, respectively (Salehi, Mirbehbahani et al. 2014).

After obtaining permission from the Ethics Committee at Ilam University of Medical Sciences, the researcher initiated the research study. The participants were told that their participation was voluntary and that all their personal information was to be kept confidential. They were also not required to write down their first and last names. Data were analyzed with the SPSS Software version 20 using t-test (for two groups of variables), ANOVA (for more than two groups of variables), and correlation analysis. To analyze the variables, the level of significance was set at $\mathrm{p} \leq 0.05$.

\section{RESULTS AND DISCUSSION}

The results showed that the majority of patients mean (SD) age of 47.46 (9.12) years, Female 143 (57.2\%), education diplomas $141(56.4 \%)$ and 500 to 1 million 116 (46.4\%).

The findings showed that a majority of participants with diabetes had marginal health literacy (40.8\%) and average general health (57.6\%) (Tables 2 and 3). The findings also indicated that the means and standard deviations of patients' health literacy scores were $31.38 \pm 6.40$, $34.93 \pm 7.45$, and $6.30 \pm 11.61$ in terms of calculations, reading skill, and general health literacy, respectively.

Table 4 shows a significant correlation between health literacy and general health status. Furthermore, it was observed that the patients with higher levels of health literacy had a better status in terms of their general health $(\mathrm{p}<0.001)$.

According to the findings, most patients with diabetes $(40.8 \%)$ had marginal health literacy, and only 27.2

\begin{tabular}{|c|c|c|}
\hline health literacy & Frequency & Percent \\
\hline inadequate & 80 & 32 \\
\hline marginal & 102 & 40.8 \\
\hline adequate & 68 & 27.2 \\
\hline Total & 250 & 100 \\
\hline
\end{tabular}

\begin{tabular}{|l|l|l|l|l|}
\hline \multicolumn{5}{|l|}{ Table 3. Frequency distribution of patients' general health scores } \\
\hline general health & adequate & average & inadequate & M(SD) \\
\hline physical health & $12(4.8)$ & $120(48)$ & $118(47.2)$ & $12.39(5.27)$ \\
\hline anxiety and insomnia & $10(4)$ & $113(45.2)$ & $127(50.8)$ & $12.88(5.33)$ \\
\hline social dysfunction & $9(3.6)$ & $0(0)$ & $241(96.4)$ & $13.60(5.26)$ \\
\hline depression & $6(2.4)$ & $0(0)$ & $244(97.6)$ & $13.49(5.12)$ \\
\hline Total & $8(3.2)$ & $144(57.6)$ & $98(39.2)$ & $52.30(18.90)$ \\
\hline
\end{tabular}


Table 4. Mean scores of health literacy based on the patients general health status

\begin{tabular}{|l|l|l|l|l|}
\hline \multicolumn{2}{|c|}{ Variable } & $\mathrm{N}(\%)$ & $\mathrm{M}(\mathrm{SD})$ & $\mathrm{p}$-value \\
\hline \multirow{3}{*}{ general health } & adequate & $8(3.2)$ & $76.50(14.88)$ & \multirow{2}{*}{.000} \\
\cline { 2 - 4 } & average & $144(57.6)$ & $69.58(10.48)$ & \\
\cline { 2 - 4 } & inadequate & $98(39.2)$ & $60.65(10.56)$ & \\
\hline
\end{tabular}

percent of these patients had adequate health literacy. This is in a similar vein to the findings of Arbabi et al. (Arbabi 2017). investigated the health literacy status in patients with diabetes referred to clinics in Zabol and concluded that the majority of patients had marginal health literacy. However, Rezai Isfahroud et al.'s study, which aimed to assess the health literacy status in Yazd Diabetes Research Centers, showed that the health literacy status was inadequate, marginal, and adequate in $59.3 \%, 18.5 \%$, and $22.2 \%$ of patients with diabetes referred to these centers, respectively(Rezaee Esfahrood, Haerian ardekani et al. 2016).

In a national study conducted by Tavousi et al. (Tavousi , Aliasghar et al. 2016)on individuals aged 18 to 65 years living in urban areas, one out of two Iranian people had limited health literacy. Using the TOFHLA, Molakhalili et al. (Mollakhalili, Papi et al. 2014)also conducted a study on 384 patients admitted in Isfahan hospitals and concluded that the mean score of health literacy was a little greater than average and that most patients had inadequate health literacy. In the study by Tehrani Banihashemi et al. (Tehrani Banihashemi, Amirkhani et al. 2007)on participants aged 18 and older in five cities located in Bushehr Province, 28.1\%, 15.3\%, and $56.6 \%$ of those surveyed had adequate, marginal, and inadequate levels of health literacy, respectively. Mozaffari et al.'s study aimed to determine the status of health literacy in parents with primary school children in Ilam using a researcher-made questionnaire. In this study, the mean scores of health literacy for fathers and mothers were $19.74 \pm 321.64$ and $14.08 \pm$ 321.71, respectively. This finding was inconsistent with the findings of the current study (Mozafari and Borji 2017).

The findings of the present study indicated a statistically significant relationship between health literacy of patients with type II diabetes and their general health status. In this regard, the findings were consistent with those of Seyedoshohadaee et al. (Seyedoshohadaee, Kaghanizade et al. 2016) and Arbabi et al. (Arbabi 2017). Conversely, Karimi et al.'s study conducted on individuals aged 18 to 64 years in Isfahan suggested no significant relationship between health literacy and general health status (Karimi, Keyvanara et al. 2014).

\section{CONCLUSION}

Given that health literacy and general health of the studied patients were not at an acceptable level, it is necessary to conduct further research to determine the effects of different training methods and nursing models toward improving the health status of patients and to provide grounds for promoting the patients' health literacy and general health status.

\section{CONFLICT OF INTEREST}

There is no conflict of interest between authors.

\section{SOURCE OF FUNDING}

Ilam University of Medical Sciences.

\section{REFERENCES}

Arbabi, H. A. M., Ali A Nooshirvani, Sajedeh A Arbab, Azadeh (2017). The Relationship Between Health Literacy and General Health in Patients with Type II Diabetes Referring to Diabetes Clinic of Zabol in 2016.Journal of Diabetes Nursing 5(1): 29-39.

Chew, L. D., K. A. Bradley, et al. (2004). Brief questions to identify patients with inadequate health literacy. Health 11: 12.

Esmaeili Shahmirzadi, S., D. Shojaeizadeh, et al. (2012). The impact of chronic diseases on the quality of life among the elderly people in the east of Tehran.Journal of Payavard Salamat 6(3): 225-235.

Javadzade, S. H., G. Sharifirad, et al. (2012). Relationship between health literacy, health status, and healthy behaviors among older adults in Isfahan, Iran." Journal of education and health promotion 1 .

Kalichman, S. C., E. Benotsch, et al. (2000). Health literacy and health-related knowledge among persons living with HIV/ AIDS. American journal of preventive medicine 18(4): 325331.

Kalichman, S. C. and D. Rompa (2000). Functional health literacy is associated with health status and health-related knowledge in people living with HIV-AIDS. Journal of acquired immune deficiency syndromes (1999) 25(4): 337-344.

Karimi, S., M. Keyvanara, et al. (2014). Health literacy, health status, health services utilization and their relationships in adults in Isfahan. Health Inf Manage 10(6): 862-875. 
Khosravi, A., K. Ahmadzadeh, et al. (2015). Health Literacy Levels of Diabetic Patients Referred to Shiraz Health Centers and Its Effective Factors. Health Information Management: 205.

Kindig, D. A., A. M. Panzer, et al. (2004). Health literacy: a prescription to end confusion, National Academies Press.

McDarbyc, J. M. and C. L. Acerinie (2014). Psychological care of children and adolescents with type 1 diabetes. Pediatric diabetes 15(20): 232-244.

Moayedi, F., S. Zare, et al. (2015). Anxiety and depression in diabetic patient referred to Bandar Abbas diabetes clinic. Bimonthly Journal of Hormozgan University of Medical Sciences 18(1): 65-71.

Mohan, V., S. Sandeep, et al. (2007). Epidemiology of type 2 diabetes: Indian scenario." Indian Journal of medical Research 125(3): 217

Mollakhalili, H., A. Papi, et al. (2014). A survey on health literacy of inpatient's educational hospitals of Isfahan University of Medical Sciences in 2012." Journal of education and health promotion 3(1): 66.

Mozafari, M. and M. Borji (2017). Assessing the health literacy level of parents in School children ilam in 2015.Journal of Nursing Education 5(6): 53-61.

Peerson, A. and M. Saunders (2009). Health literacy revisited: what do we mean and why does it matter?" Health promotion international 24(3): 285-296.

Rezaee Esfahrood, Z., A. Haerian ardekani, et al. (2016). A Survey on Health Literacy of Referred Diabetic Patients to
Yazd Diabetes Research Center. Tolooebehdasht 15(3): 176186.

Salehi, M., N. Mirbehbahani, et al. (2014). General health of beta-thalassemia major patients in Gorgan, Iran.Journal of Gorgan University of Medical Sciences 16(1): 120-125.

Schillinger, D., K. Grumbach, et al. (2002). Association of health literacy with diabetes outcomes. Jama 288(4): 475-482.

Seyedoshohadaee, M., M. Kaghanizade, et al. (2016). The Relationship Between Health Literacy And General Health In Patients With Type 2 Diabetes. Iranian Journal of Diabetes and Metabolism 15(5): 312-319.

Sihota, S. and L. Lennard (2004). Health literacy: being able to make the most of health, National Consumer Council.

Tavousi , M., H. M. ALIASGHAR, et al. (2016). Health Literacy In Iran: Findings From A National Study."

Tehrani Banihashemi, S.-A., M. A. Amirkhani, et al. (2007) Health literacy and the influencing factors: a study in five provinces of Iran. Strides in Development of Medical Education 4(1): 1-9.

Varvani Farahani, P., M. R. Rezvanfar, et al. (2016). Comparing The Effect Of Multimedia Education With Live Successful Experiments On Quality Of Life In Type 2 Diabetic Patients." Iranian Journal of Diabetes and Metabolism 15(5): 320329.

Williams, M. V., D. W. Baker, et al. (1998). Relationship of functional health literacy to patients' knowledge of their chronic disease: a study of patients with hypertension and diabetes. Archives of internal medicine 158(2): 166-172. 